\title{
Carrier frequency of the GJB2 mutations that cause hereditary hearing loss in the Japanese population
}

\begin{abstract}
Mirei Taniguchi ${ }^{1}$, Hirotaka Matsuo ${ }^{2}$, Seiko Shimizu², Akiyoshi Nakayama ${ }^{2}$, Koji Suzuki ${ }^{3}$, Nobuyuki Hamajima ${ }^{4}$, Nariyoshi Shinomiya ${ }^{2}$, Shinya Nishio ${ }^{5}$, Shinji Kosugi ${ }^{6}$, Shin-ichi Usami ${ }^{5}$, Juichi Ito ${ }^{1}$ and Shin-ichiro Kitajiri ${ }^{1}$

Hearing impairment is one of the most common sensory disorders that affect $\sim 1$ in 1000 children, and half of them are considered to be hereditary. Information about the carrier frequencies of mutations that underlie autosomal recessive disorders is indispensable for accurate genetic counseling to predict the probability of patients' children's disease. However, there have been few reports specific to the Japanese population. GJB2 mutations are reported to be the most frequent cause of hereditary hearing loss, and the mutation spectrum and frequency of GJB2 mutations were reported to vary among different ethnic groups. In this study, we investigated the carrier frequency of GJB2 mutations and the mutation spectrum in 509 individuals randomly selected from the general Japanese population. We show that the carrier frequencies of the two most common pathogenic mutations are $1.57 \%(8 / 509)$ for c.235delC and $1.77 \%(9 / 509)$ for p.Val37lle. In addition to these mutations, we found two pathogenic variants (p.[Gly45Glu;Tyr136*] and p.Arg143Trp), and the total carrier frequency was estimated to be around 3.73\% (19/509). We also detected six unclassified variants, including two novel variants ( $p$. Cys60Tyr and p.Phe106Leu), with the former predicted to be pathogenic. These findings will provide indispensable information for genetic counseling in the Japanese population.
\end{abstract} Journal of Human Genetics (2015) 60, 613-617; doi:10.1038/jhg.2015.82; published online 16 July 2015

\section{INTRODUCTION}

Inherited diseases are frequently found worldwide and a major group of inherited diseases is autosomal recessive disorders such as non-syndromic hereditary hearing impairment, Fukuyama congenital muscular dystrophy, Wilson disease and xeroderma pigmentosum. The carrier frequencies of these diseases are estimated to be $0.9 \%$ for Fukuyama congenital muscular dystrophy, ${ }^{1} 1.1 \%$ for Wilson disease ${ }^{2}$ and $0.9 \%$ for xeroderma pigmentosum (see the Japan Intractable Disease Information Center's website: http://www.nanbyou.or.jp/ entry/112). The mutation spectrum of these diseases is reported to be population specific. ${ }^{1,3}$ Among these autosomal recessive disorders, non-syndromic congenital hearing impairment is reported to be the most frequent. $^{4-6}$ Approximately 1 in 1000 new-born children are affected by congenital hearing impairment, and 50-60\% cases of them are attributable to genetic causes. ${ }^{7}$ Hereditary hearing loss has considerable genetic heterogeneity and, to date, more than 100 genetic loci have been discovered to be the cause. ${ }^{8}$ Genetic testing for congenital hearing impairment has become more important for accurate diagnoses, prediction of severity of hearing loss, estimation of associated abnormalities, selection of appropriate habilitation options, prevention of hearing loss and better genetic counseling. ${ }^{9}$ Recently, the panel screening of multiple mutations by invader assay ${ }^{10}$ was reported to be very efficient. ${ }^{9}$ Genetic testing by invader assay is now performed throughout Japan, and the Japanese National Health Insurance began to cover its costs from April 2012. Currently, hearingimpaired patients can easily access clinical diagnostic screening by genetic testing, which should be accompanied by the appropriate genetic counseling that includes an estimation of the next generation's incidence (or recurrence) of hearing difficulty.

Most hereditary impairment is caused by recessive mutations in genes such as GJB2 and SLC26A4. ${ }^{7}$ In the case of autosomal recessive disorders, patients have mutations in both the paternal and maternal alleles. Therefore, to estimate the next generation's incidence ratio, the carrier frequencies of the mutations in these genes must be ascertained. However, there are only a limited number of reports about the carrier frequencies of autosomal recessive disorders, such as hearing impairment, in the Japanese population. ${ }^{5,6}$

The GJB2 gene is the most prevalent for congenital hearing loss ${ }^{11,12}$ and the mutation spectrums are known to vary among different ethnic groups. ${ }^{6}$ It is vital to study the carrier frequency and mutation spectrum of each genetic background to enable more precise genetic counseling. The GJB2 gene encodes connexin26, a member of the connexin family of proteins that are constituents of the intercellular gap junction. ${ }^{13}$ The first GJB2 mutation was reported by Kelsell et al.

${ }^{1}$ Department of Otolaryngology, Head and Neck Surgery, Graduate School of Medicine, Kyoto University, Kyoto, Japan; ${ }^{2}$ Department of Integrative Physiology and Bio-Nano Medicine, National Defense Medical College, Tokorozawa, Japan; ${ }^{3}$ Department of Public Health, Fujita Health University School of Medicine, Toyoake, Japan; ${ }^{4}$ Department of Healthcare Administration, Nagoya University Graduate School of Medicine, Nagoya, Japan; ${ }^{5}$ Department of Otorhinolaryngology, Shinshu University School of Medicine, Matsumoto, Japan and ${ }^{6}$ Department of Medical Ethics/Medical Genetics, Kyoto University School of Public Health, Kyoto, Japan

Correspondence: Dr S Kitajiri, Department of Otolaryngology, Head and Neck Surgery, Graduate School of Medicine, Kyoto University, Kawaharacho 54, Shogoin, Sakyo-ku, Kyoto 606-8507, Japan.

E-mail: kitajirient.kuhp.kyoto-u.ac.jp

Received 12 February 2015; revised 22 June 2015; accepted 11 June 2015; published online 16 July 2015 
Table 1 GJB2 variants detected in 509 Japanese people

\begin{tabular}{|c|c|c|c|c|c|c|c|}
\hline Codon & Nucleotide & Heterozygote & Homozygote & $\begin{array}{l}\text { Frequency of mutant } \\
\text { alleles }(\mathrm{n}=1018)\end{array}$ & $\begin{array}{l}\text { Number of persons with } \\
\text { mutations }(\mathrm{n}=509)\end{array}$ & $\begin{array}{l}\text { Characterization of } \\
\text { variant }\end{array}$ & Reference \\
\hline p.T8M & c. $23 \mathrm{C}>\mathrm{T}$ & 2 & 0 & $0.20 \%$ & $2(0.39 \%)$ & $\begin{array}{l}\text { Unproven pathogenic } \\
\text { nature }\end{array}$ & Kenna et al. ${ }^{26}$ \\
\hline p.G12fs ${ }^{a}$ & c.35delG & 0 & 0 & 0 & 0 & Pathogenic & $\begin{array}{l}\text { Denoyelle et al.. }{ }^{28} \text { Estivill } \\
\text { et al. }{ }^{22}\end{array}$ \\
\hline p.V27I & c. $79 \mathrm{G}>\mathrm{A}$ & 235 & 71 & $37.00 \%$ & $306(60.12 \%)$ & Not pathogenic & Zelante et al., ${ }^{11}$ Abe et al. ${ }^{5}$ \\
\hline p.L56fs & c.167delT & 0 & 0 & 0 & 0 & Pathogenic & Zelante et al. ${ }^{11}$ \\
\hline p.C60Y & c. $179 \mathrm{G}>\mathrm{A}$ & 1 & 0 & $0.10 \%$ & $1(0.20 \%)$ & $\begin{array}{l}\text { Unproven pathogenic } \\
\text { nature }\end{array}$ & This study \\
\hline p.V63V & c. $189 \mathrm{G}>\mathrm{T}$ & 1 & 0 & $0.10 \%$ & $1(0.20 \%)$ & $\begin{array}{l}\text { Not pathogenic } \\
\text { (silent variation) }\end{array}$ & This study \\
\hline p.R127H & c. $380 \mathrm{G}>\mathrm{A}$ & 1 & 0 & $0.10 \%$ & $1(0.20 \%)$ & $\begin{array}{l}\text { Unproven pathogenic } \\
\text { nature }\end{array}$ & $\begin{array}{l}\text { Estivill et al., }{ }^{22} \text { Roux } \\
\text { et al. }{ }^{27}\end{array}$ \\
\hline p.E114G & c. $341 \mathrm{~A}>\mathrm{G}$ & 118 & 8 & $13.20 \%$ & $126(24.75 \%)$ & Not pathogenic & Green et al., ${ }^{15}$ Fuse et al..$^{20}$ \\
\hline p.T123N & c. $368 \mathrm{C}>\mathrm{A}$ & 6 & 0 & $0.59 \%$ & $6(1.18 \%)$ & Not pathogenic & $\begin{array}{l}\text { Park et al., }{ }^{30} \text { Abe et al., }{ }^{5} \\
\text { Ohtsuka et al. }{ }^{6}\end{array}$ \\
\hline p.R143Wa & c. $427 \mathrm{C}>\mathrm{T}$ & 1 & 0 & $0.10 \%$ & $1(0.20 \%)$ & Pathogenic & Brobby et al., 31 \\
\hline p.F191L $L^{a, b}$ & c. $570 \mathrm{~T}>\mathrm{C}$ & 1 & 0 & $0.10 \%$ & $1(0.20 \%)$ & $\begin{array}{l}\text { Unproven pathogenic } \\
\text { nature }\end{array}$ & Ohtsuka et al. ${ }^{6}$ \\
\hline p.1203T & c. $608 \mathrm{~T}>\mathrm{C}$ & 55 & 1 & $5.60 \%$ & $56(11.0 \%)$ & Not pathogenic & Kudo et al., ${ }^{21}$ Abe et al. ${ }^{5}$ \\
\hline
\end{tabular}

We detected nine pathogenic mutant alleles that had p.V37I, eight c.235delC, one p.[G45E;Y136X] and one p.R143W. We also detected two p.T8M, one p.I71T, one p.R127H and one p.F191L mutation, the pathogenesis of which had not been proven. GJB2 carrier frequency in Japanese people was estimated to be $19 / 509$ (3.73\%).

aMutations screened by Invader assay covered by the Japanese National Health Insurance (Abe et al. ${ }^{10}$ ).

${ }^{b}$ Variants possibly representing non-pathogenic polymorphisms (Ohtuska et al. ${ }^{6}$ ).

in $1997^{14}$ and since then more than 100 pathogenic variants have been reported worldwide (see the Connexin-deafness website: http:// davinci.crg.es/deafness/). In a previous report, GJB2 mutations were estimated to be responsible for about $15-20 \%$ of the cases of deafness ${ }^{7}$ in the US, and $12.5 \%(33 / 264)$ of those in Japan. ${ }^{9}$ The c.35delG mutation is the most common in the Caucasian population, and the c.235delC and p.Val37lle mutations are the most prevalent in the Asian population. ${ }^{6,15}$ The carrier frequency of GJB2 mutations in the control population is reported to be $2-4 \%{ }^{15-17}$ in the US, in Europe, and in Korea, but the carrier frequency in the Japanese population has not yet been reported. The information about both the carrier frequency and spectrum with respect of GJB2 mutations in Japan is very important because GJB2 mutations were reported to have a phenotype-genotype correlation. ${ }^{18,19}$

The purpose of this study is to investigate the carrier frequency of GJB2 mutations and the mutation spectrum in the Japanese population to provide more accurate information for genetic counseling. This is the first study in Japan to estimate the carrier frequency of the GJB2 mutations causing hereditary hearing loss, the most frequent autosomal recessive disorder in Japan.

\section{SUBJECTS AND METHODS}

Experiments were approved by the Kyoto University Graduate School and Faculty of Medicine, Ethics Committee (Permit number: G294), and written informed consent was obtained from each participant prior to genetic analysis. Blood samples were collected from 509 healthy Japanese people (201 males and 308 females) at annual health checks in Yakumo, Hokkaido in Japan. Their ages ranged from 40 to 91 years, and the average was 67.8 years. According to the questionnaire filled in by the subjects, $56 / 509$ (11.0\%) had subjectively experienced hearing difficulty, and in terms of vestibular function, 106/509 (20.8\%) had experienced vertigo or dizziness. From the blood sample, we measured HbAlc (NGSP; National Glycohemoglobin Standardization Program) levels and 36 out of 509 people (7.1\%) were over 6.5 , revealing a risk of diabetes, which is compatible with Japanese general population. The result from our questionnaire are consistent with Japan Trak report in which $10.8 \%$ of the randomly selected 15036 respondents reported experiencing hearing difficulty (see the Japan Trak website: http://www.atpress. ne.jp/view/32264). Therefore, the subjects enrolled in this study are thought to be representative of the population in Japan.

To identify GJB2 mutations, a fragment containing the entire coding region were amplified from genomic DNA sample by PCR using the primer pair 5'-GGTGAGGTTFTFTAAGAGTTGG-3'/5'-CTACGGTGTTACTCGAGACGA-3', and the amplicon were used as a template for direct sequencing. ${ }^{5}$ Direct sequencing analysis was performed using an ABI PRISM Genetic Analyzer (Applied Biosystems, Waltham, MA, USA), and the data were analyzed using ABI Variant Reporter v1.1 (Applied Biosystems).

\section{RESULTS}

Table 1 summarizes the variants identified in this study. Among the mutations screened by the invader assay, ${ }^{9}$ which are covered by Japanese health insurance, we found six previously reported pathogenic variants within the coding region of GJB2. In detail, we detected 
nine alleles for p.Val37Ile, eight for c.235delC, one for p.[Gly45Glu; Tyr136*], one for p.Arg143Trp, one for p.Ile71Thr and one for p.Phe191Leu; p.Gly45Glu and p.Tyr $136^{*}$ mutations are on the same parental allele. ${ }^{19}$ Among these mutations, the pathogenicity of p.Ile71Thr and p.Phe191Leu has been controversial, and they are considered to represent non-pathogenic polymorphisms. ${ }^{6} \mathrm{We}$ also found previously reported variants, p.Thr8Met and p.Arg127His, and two novel variants, p.Cys60Tyr and p.Phe106Leu, which were not included in the Invader assay-based mutation screening. The pathogenicity of p.Thr8Met, and p.Arg127His variants is unknown.

We found 19 previously reported pathogenic variants, and 5 variants found in this study were unclassified, so the carrier frequency of the GJB2 mutations is estimated to be at least $3.73-4.72 \%$ (19-24/509). We believe that the screening (including the four variants, p.Thr8Met, p.Ile71Thr, p.Arg127His and p.Phe191Leu, and other novel variants) will be very important for more accurate genetic testing. The pathogenicity of novel variants is not clear and requires further studies for clarification.

The two novel variants mentioned above, p.Cys60Tyr and p. Phe106Leu, and one silent variant, p.Val63Val (c.189G $>$ T), were identified in this study. Figure 1 shows amino acid sequences of Homo sapiens and other species' connexin26 and the two novel variants, p.Cys60Tyr and p.Phe106Leu. Cys60 is evolutionarily conserved, while the leucine residue of p.Phe106Leu is found in Xenopus laevis. To predict the effect of the variants, we carried out computer analysis using the Sorting Intolerant from Tolerant (SIFT: http://sift.jcvi.org/) and Polymorphism Phenotyping (PolyPhen2: http://genetics.bwh. harvard.edu/pph2/). The functional prediction of p.Cys60Tyr was Damaging (1.0) by SIFT, and Damaging (1.0) by PolyPhen2. On the other hand, that of p.Phe106Leu was Tolerated (0.23) by SIFT and Benign (0) by PolyPhen2. Considering these data, p.Cys60Tyr is expected to be pathogenic and p.Phe106Leu to be a non-pathogenic polymorphism.

Synonymous variants are usually non-pathogenic unless the nucleotide change alters the splicing of the RNA. Analysis of the p.Val63Val (c.189G $>$ T) synonymous variant using the prediction program

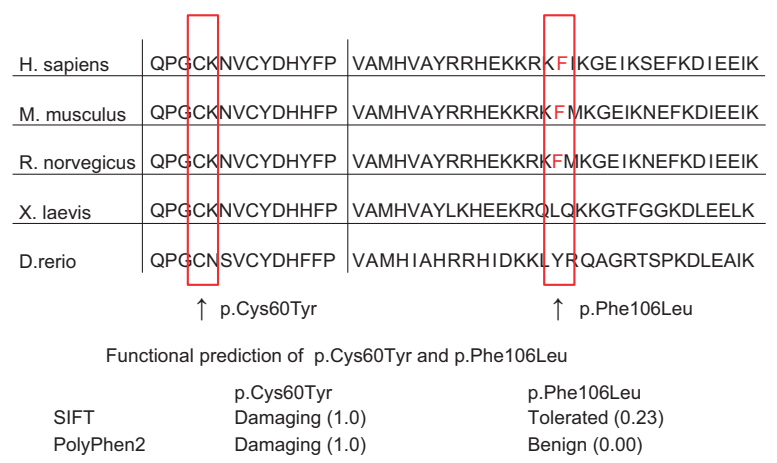

Figure 1 Alignment of amino-acid sequences of human and other species' connexin26 (Cx26) showing the mutation sites of two novel variants, p.Cys60Tyr and p.Phe106Leu, and functional prediction of p.Cys60Tyr and p.Phe106Leu. We found two novel variants p.Cys60Tyr and p.Phe106Leu. To predict their pathogenesis, we compared amino-acid sequences of human and other species' Cx26. As Cys60 is evolutionarily conserved, p.Cys60Tyr could be pathogenic; p.Phe106Leu is probably a non-pathogenic polymorphism because Xenopus has a leucine at this position. The functional prediction of p.Cys60Tyr was Damaging (1.0) by SIFT, and Damaging (1.0) by PolyPhen2. That of p.Phe106Leu was Tolerated (0.23) by SIFT and Benign (0) by PolyPhen2. Prediction by SIFT and PolyPhen2 also showed that p.Cys60Tyr would be pathogenic and p.Phe106Leu would be a non-pathogenic polymorphism.
NNSPLICE (http://www.fruitfly.org/seq_tools/splice.html) indicates that this variant is not expected to be either a donor or an acceptor site for splicing and is, therefore, likely to be non-pathogenic. The three subjects carrying p.Cys60Tyr, p.Phe106Leu or p.Val63Val had no experience of hearing difficulty or tinnitus.

We found six alleles of the p.Thr123Asn mutation, but we did not consider them to be pathogenic variants; p.Thr123Asn has been considered to be a rare non-pathogenic variant because it was found with equal frequency in patients and controls in Ohtsuka. ${ }^{6}$ In contrast, the non-pathogenic variants, p.Val27Ile, p.Glu114Gly and p.Ile203Thr, ${ }^{11,15,20,21}$ that are frequently found in the Japanese population ${ }^{6}$ were also frequently observed in this study. The most common GJB2 mutation in Caucasian people, the c.35delG mutation, was not found in these subjects, indicating differing variants among ethnic groups. All things considered, the GJB2 carrier frequency in the Japanese population was estimated to be at least 19/509 (3.73\%). We did not find any subjects who carry homozygous or compound heterozygous pathogenic mutations in this study.

\section{DISCUSSION}

There are several reports on carrier frequency and the mutation spectrum of GJB2 mutations. In Europe, the carrier frequency of c.35delG was reported to be around 2-3\%. ${ }^{16,22,23}$ Green et al., ${ }^{15}$ reported that 14 out of 560 general people carried the c.35delG variant, so they estimated that the carrier rate of c.35delG was about $2.5 \%$ in the Midwestern USA.

In the Ashkenazi Jewish population, it was reported that the carrier frequency of c.167delT variant was $4.03 \%(22 / 546) .{ }^{24}$ In the Thai population, p.Val37Ile was the most frequent variant and the carrier frequency of this variant was $17.1 \%(35 / 205)^{25}$ in healthy controls. In the Korean population, the carrier frequency of p.Val37Ile was $1.35 \%$ (28/2072), and that of c.235delC was $1.25 \%$ (26/2072), and no c.35delG mutants were detected in their study. ${ }^{17}$

The majorities of studies have focused only on hearing-impaired patients and their allele frequency and have not focused on the carrier frequency. Ohtsuka et al. ${ }^{6}$ reported that the frequency of the mutant alleles among hearing-impaired patients was 3.9\% (96/2454) for the c.235delC mutation, and 2.5\% (61/2454) for the p.Val37Ile mutation. Tsukada et al. ${ }^{19}$ reported that the mutation spectrum and frequency in a large number of Japanese hearing-impaired patients, and the c.235delC mutation allele frequency was 5.3\% (142/2686), and that of p.Val37lle was $1.8 \%(47 / 2686)$.

In this study, we found eight alleles of the c.235delC mutant $(8 / 1018,0.79 \%)$ and nine alleles of the p.Val37Ile mutant $(9 / 1018$, $0.88 \%$ ) in the control population. Based on our observation, the carrier frequency of c.235delC was $1.57 \%$ (8/509), and that of p.Val37Ile was $1.77 \%(9 / 509)$. In addition to these two most common mutations, we found two other pathogenic variants (p.[Gly45Glu;Tyr136*], and p. Arg143Trp) in this study, and the carrier frequency was estimated to be around $3.73 \%$ (19/509). Furthermore, we identified two novel variants (p.Cys60Tyr and p.Phe106Leu) whose pathogenic nature is unknown. Amino-acid conservation information and prediction by computer analysis (SIFT and PolyPhen2) indicated that p.Cys60Tyr may be pathogenic and that p.Phe106Leu would be a non-pathogenic polymorphism. In addition to these prediction method, functional studies would shed light on the pathogenesis on these variants.

In this study, we also detected two p.Thr8Met variants, one p.Ile71Thr variant, one p.Arg127His variant and one p.Phe191Leu variant, all with uncertain pathogenicity; p.Ile71Thr and p.Phe191Leu were first reported in Japanese patients. ${ }^{6}$ Ohtsuka et al. ${ }^{6}$ found four p.Ile71Thr and four p.Phe191Leu variants in 2454 alleles, but 
p.Ile71Thr and p.Phe191Leu were only found to be heterozygous with no other mutations being detectable; therefore, it remains a possibility that they were non-pathogenic rare polymorphisms. To date, the pathogenicity of p.Ile71Thr and p.Phe191Leu mutations has not been proven, ${ }^{6,19}$ so they may be non-pathogenic variants. The functional prediction of p.Ile71Thr was Damaging (1.0) by SIFT and Damaging (0.972) by PolyPhen2. That of p.Phe191Leu was Damaging (0.99) by SIFT and Damaging (1.0) by PolyPhen2. The p.Thr8Met variant was first reported by Kenna et al. ${ }^{26}$ from hearing loss patients in the US population, and it was also reported to be found in hearing-impaired patients in Japan.9,19 The functional prediction of p.Thr8Met was Damaging $(0.97)$ by SIFT and Possibly Damaging (0.775) by PolyPhen2. The pathogenesis of the p.Arg127His variant is controversial $^{17}$ as this variant was detected in one family with congenital deafness ${ }^{22}$ but the variant was found in a homozygous state in individuals with normal hearing in another study. ${ }^{27}$ In addition this variant was predicted to be Tolerated (0.85) by SIFT and Benign $(0.001)$ by PolyPhen2. Considering these prediction analyses, p.Thr8Met, p.Ile71Thr and p.Phe191Leu could be pathogenic.

The carrier frequency and mutation spectrum of the Japanese population was different from those of Caucasian populations; the carrier frequency of c.35delG and c.235delC mutation is reported to be $2-3 \%$ and $0 \%$ in Caucasian, ${ }^{15,16,22,23}$ whereas $0 \%$ and $1.57 \%$ in the present study, respectively. In addition, p.Val37Ile is revealed to be common in Asian countries; the carrier frequency was $1.77 \%$ in Japanese, $1.35 \%$ in Korea and $17.1 \%$ in Thai population, respectively. ${ }^{17,25}$ In patients with impaired hearing, GJB2 mutations were reported to have a phenotype-genotype correlation. ${ }^{18,19}$ For example, the c.235delC homozygous mutation and the c.235delC/p. Arg143Trp compound heterozygous mutation show severe hearing impairment, while the p.Val37Ile homozygous mutation has a mild phenotype. ${ }^{19}$

Among hearing-impaired patients in Japan, the c.235delC mutation is reported to be the most prevalent mutation. ${ }^{5,6,20}$ However, in people with normal hearing, the p.Val37Ile mutation was more frequent than c. 235 delC in our study, as with previous studies. ${ }^{19}$ This apparent contradiction may be because patients with the p.Val37Ile mutation have a milder phenotype and may therefore be excluded from the research on hearing loss. ${ }^{19}$

Generally, in the case of autosomal recessive diseases, if the parents are related, the probability of a homozygous mutant allele increases. However, even if the parents are not related, it is still possible for both to be GJB2 mutation carriers because the GJB2 carrier frequency is estimated to be as high as around $3.7 \%$.

In conclusion, we estimate that the GJB2 carrier frequency of the Japanese population would be about $3.7 \%$. Almost half of these would have the p.Val37lle mutation, whereas individuals homozygous for this mutation have a relative mild phenotype. The other major mutation allele would be c.235delC. Our study is the latest and the largest report on carrier frequencies of GJB2 mutations in the Japanese general population to date. This is indispensable information for predicting the next generation's incidence of hearing difficulty. We believe that information from this study will be useful in genetic counseling in Japan.

\section{ACKNOWLEDGEMENTS}

This study was supported by a Health and Labor Sciences Research Grant from the Ministry of Health, Labor and Welfare of Japan to JI. We would like to thank Dr Hiroki Inoue for collection of samples and also for technical assistance.
1 Kato, R., Kawamura, J., Sugawara, H., Niikawa, N. \& Matsumoto, N. A rapid diagnostic method for a retrotransposal insertional mutation into the FCMD gene in Japanese patients with Fukuyama congenital muscular dystrophy. Am. J. Med. Genet. 127A, 54-57 (2004).

2 Kusuda, Y., Hamaguchi, K., Mori, T., Shin, R., Seike, M. \& Sakata, T. Novel mutations of the ATP7B gene in Japanese patients with Wilson disease. J. Hum. Genet. 45 86-91 (2000).

3 Shimizu, N., Nakazono, H., Takeshita, Y., Ikeda, C., Fujii, I., Watanabe, A. et al. Molecular analysis and diagnosis in Japanese patients with Wilson's disease. Pediatr. Int. 41, 409-413 (1999).

4 Song, M. J., Lee, S. T., Lee, M. K., Ji, Y., Kim, J. W. \& Ki, C. S. Estimation of carrier frequencies of six autosomal-recessive Mendelian disorders in the Korean population. J. Hum. Genet. 57, 139-144 (2012).

5 Abe, S., Usami, S., Shinkawa, H., Kelley, P. M. \& Kimberling, W. J. Prevalent connexin 26 gene (GJB2) mutations in Japanese. J. Med. Genet. 37, 41-43 (2000)

6 Ohtsuka, A., Yuge, I., Kimura, S., Namba, A., Abe, S., Laer, L. V. et al. GJB2 deafness gene shows a specific spectrum of mutations in Japan, including a frequent founder mutation. Hum. Genet. 112, 329-333 (2003).

7 Morton, C. C. \& Nance, W. E. Newborn hearing screening-a silent revolution. N. Engl. J. Med. 354, 2151-2164 (2006).

8 Steel, K. P. \& Kros, C. J. A genetic approach to understanding auditory function. Nat. Genet. 27, 143-149 (2001)

9 Usami, S., Nishio, S., Nagano, M., Abe, S. \& Yamaguchi, T.Consortium, D.G.S. Simultaneous screening of multiple mutations by invader assay improves molecular diagnosis of hereditary hearing loss: a multicenter study. PLoS One 7, e31276 (2012).

10 Abe, S., Yamaguchi, T. \& Usami, S. Application of deafness diagnostic screening panel based on deafness mutation/gene database using invader assay. Genet. Test. 11, 333-340 (2007).

11 Zelante, L., Gasparini, P., Estivill, X., Melchionda, S., D’Agruma, L., Govea, N. et al. Connexin26 mutations associated with the most common form of non-syndromic neurosensory autosomal recessive deafness (DFNB1) in Mediterraneans. Hum. Mol Genet. 6, 1605-1609 (1997).

12 Smith, R. J., Bale, J. F. \& White, K. R. Sensorineural hearing loss in children. Lancet $365,879-890$ (2005)

13 Kumar, N. M. \& Gilula, N. B. The gap junction communication channel. Cell 84 381-388 (1996).

14 Kelsell, D. P., Dunlop, J., Stevens, H. P., Lench, N. J., Liang, J. N., Garry, G. et al. Connexin 26 mutations in hereditary non-syndromic sensorineural deafness. Nature 387, 80-83 (1997).

15 Green, G. E., Scott, D. A., McDonald, J. M., Woodworth, G. C., Sheffield, V. C. \& Smith, R. J.H. Carrier rates in the midwestern United States for GJB2 mutations causing inherited deafness. JAMA 281, 2211-2216 (1999)

16 Lucotte, G. \& Mercier, G. Meta-analysis of GJB2 mutation 35delG frequencies in Europe. Genet. Test 5, 149-152 (2001).

17 Han, S. H., Park, H. J., Kang, E. J., Ryu, J. S., Lee, A., Yang, Y. H. et al. Carrie frequency of GJB2 (connexin-26) mutations causing inherited deafness in the Korean population. J. Hum. Genet. 53, 1022-1028 (2008).

18 Oguchi, T., Ohtsuka, A., Hashimoto, S., Oshima, A., Abe, S., Kobayashi, Y. et al. Clinical features of patients with GJB2 (connexin 26) mutations: severity of hearing loss is correlated with genotypes and protein expression patterns. J. Hum. Genet. 50, 76-83 (2005).

19 Tsukada, K., Nishio, S. \& Usami, S.Consortium, D.G.S.. A large cohort study of GJB2 mutations in Japanese hearing loss patients. Clin. Genet. 78 464-470 (2010).

20 Fuse, Y., Doi, K., Hasegawa, T., Sugii, A., Hibino, H. \& Kubo, T. Three novel connexin26 gene mutations in autosomal recessive non-syndromic deafness. Neuroreport 10, 1853-1857 (1999).

21 Kudo, T., Ikeda, K., Kure, S., Matsubara, Y., Oshima, T., Watanabe, K et al. Novel mutations in the connexin 26 gene (GJB2) responsible for childhood deafness in the Japanese population. Am. J. Med. Genet. 90, 141-145 (2000).

22 Estivill, X., Fortina, P., Surrey, S., Rabionet, R., Melchionda, S., D'Agruma, L. et al. Connexin-26 mutations in sporadic and inherited sensorineural deafness. Lancet 351 , 394-398 (1998).

23 Gasparini, P., Rabionet, R., Barbujani, G., Melchionda, S., Petersen, M. Brøndum-Nielsen, $K$. et al. High carrier frequency of the $35 \mathrm{del} G$ deafness mutation in European populations. Genetic Analysis Consortium of GJB2 35delG. Eur. J. Hum. Genet. 8, 19-23 (2000).

24 Morell, R. J., Kim, H. J., Hood, L. J., Goforth, L., Friderici, K., Fisher, R. et al. Mutations in the connexin 26 gene (GJB2) among Ashkenazi Jews with nonsyndromic recessive deafness. N. Engl. J. Med. 339, 1500-1505 (1998).

25 Wattanasirichaigoon, D., Limwongse, C., Jariengprasert, C., Yenchitsomanus, P. T., Tocharoenthanaphol, C., Thongnoppakhun, W. et al. High prevalence of V37I genetic variant in the connexin-26 (GJB2) gene among non-syndromic hearingimpaired and control Thai individuals. Clin. Genet. 66, 452-460 (2004).

26 Kenna, M. A., Wu, B. L., Cotanche, D. A., Korf, B. R. \& Rehm, H. L. Connexin 26 studies in patients with sensorineural hearing loss. Arch. Otolaryngol. Head Neck Surg. 127, 1037-1042 (2001).

27 Roux, A. F., Pallares-Ruiz, N., Vielle, A., Faugère, V., Templin, C., Leprevost, D. et al. Molecular epidemiology of DFNB1 deafness in France. BMC Med. Genet. 5 , 5 (2004). 
28 Denoyelle, F., Weil, D., Maw, M. A., Wilcox, S. A., Lench, N. J., Allen-Powell, D. R. et al. Prelingual deafness: high prevalence of a 30delG mutation in the connexin 26 gene. Hum. Mol. Genet. 6, 2173-2177 (1997).

29 Wilcox, S. A., Saunders, K., Osborn, A. H., Arnold, A., Wunderlich, J., Kelly, T. et al. High frequency hearing loss correlated with mutations in the GJB2 gene. Hum. Genet. 106, 399-405 (2000).

30 Park, H. J., Hahn, S. H., Chun, Y. M., Park, K. \& Kim, H. N. Connexin26 mutations associated with nonsyndromic hearing loss. Laryngoscope. 110, 1535-1538 (2000).

31 Brobby, G. W., Müller-Myhsok, B. \& Horstmann, R. D. Connexin 26 R143W mutation associated with recessive nonsyndromic sensorineural deafness in Africa. N. Engl. J. Med. 338, 548-550 (1998). (c) (i) (5) (2) This work is licensed under a Creative Commons Attribution-NonCommercial-ShareAlike 4.0 International License. The images or other third party material in this article are included in the article's Creative Commons license, unless indicated otherwise in the credit line; if the material is not included under the Creative Commons license, users will need to obtain permission from the license holder to reproduce the material. To view a copy of this license, visit http://creativecommons.org/licenses/ by-nc-sa/4.0/ 